\title{
A new iteration method for variational inequalities on the set of common fixed points for a finite family of quasi-pseudocontractions in Hilbert spaces
}

Haiyun Zhou ${ }^{1,2}$ and Peiyuan Wang ${ }^{1 *}$

\author{
"Correspondence: \\ wangpy629@163.com \\ 'Department of Mathematics, \\ Shijiazhuang Mechanical \\ Engineering College, Shijiazhuang, \\ 050003, China \\ Full list of author information is \\ available at the end of the article
}

\begin{abstract}
In this paper, we propose a new iteration method based on the hybrid steepest descent method and Ishikawa-type method for seeking a solution of a variational inequality involving a Lipschitz continuous and strongly monotone mapping on the set of common fixed points for a finite family of Lipschitz continuous and quasi-pseudocontractive mappings in a real Hilbert space.

MSC: Primary 41A65; 47H17; secondary 47J20
\end{abstract}

Keywords: variational inequalities; hybrid steepest descent method; quasi-pseudocontractions; strongly monotone mappings

\section{Introduction and preliminaries}

Let $C$ be a nonempty closed and convex subset of a real Hilbert space $H$ with the inner product and induced norm $\|\cdot\|$. A mapping $F$ of $C$ into $H$ is said to be monotone if

$$
\langle F x-F y, x-y\rangle \geq 0
$$

for all $x, y \in C$.

The variational inequality problem with respect to $F$ and $C$ is to find a point $z \in C$ such that

$$
\langle F z, y-z\rangle \geq 0 \quad \text { for all } y \in C
$$

Variational inequalities were initially investigated by Kinderlehrer and Stampacchia in [1], and have been widely studied by many authors ever since, due to the fact that they cover as diverse disciplines as partial differential equations, optimization, optimal control, mathematical programming, mechanics and finance (see [1-3]).

We know that if $F$ is a $k$-Lipschitz continuous and $\eta$-strongly monotone mapping, i.e., $F$ enjoys the following properties:

$$
\|F x-F y\| \leq k\|x-y\| \quad \text { and } \quad\langle F x-F y, x-y\rangle \geq \eta\|x-y\|^{2}
$$


for all $x, y \in C$, where $k$ and $\eta$ are fixed positive numbers, then (1.2) has a unique solution. It is well known that (1.2) is equivalent to the fixed point equation

$$
z=P_{C}[(I-\mu F) z]
$$

where $P_{C}$ stands for the metric projection from $H$ onto $C$ and $\mu$ is an arbitrarily positive number. Consequently, the well-known iterative procedure, the projected gradient method (PGM) [3-6], can be used to solve (1.2). PGM generates an iterative sequence by the recursion

$$
x_{1} \in C \quad \text { and } \quad x_{n+1}=P_{C}\left[(I-\mu F) x_{n}\right], \quad n \geq 1 .
$$

When $F$ is a $k$-Lipschitz continuous and $\eta$-strongly monotone mapping, as $\mu \in\left(0, \frac{2 \eta}{k^{2}}\right)$, the sequence $\left\{x_{n}\right\}$ generated by (1.4) converges strongly to a unique solution of (1.2).

The projected gradient method (1.4) involves the metric projection $P_{C}$. In order to reduce the complexity caused by $P_{C}$, Yamada [7] introduced a hybrid steepest descent method (HSDM) for solving (1.2). By assuming that $C=\bigcap_{i=1}^{N} \operatorname{Fix}\left(T_{i}\right) \neq \varnothing$, where $\operatorname{Fix}\left(T_{i}\right)=$ $\left\{x \in H: x=T_{i} x\right\}$ and $T_{i}$ is a nonexpansive mapping on $H$, i.e.,

$$
\|T x-T y\| \leq\|x-y\|
$$

for all $x, y \in H$, Yamada proposed the following iterative algorithm:

$$
x_{1} \in H, \quad x_{n+1}=\left(I-\lambda_{n} \mu F\right) T_{[n]} x_{n},
$$

where $T_{[n]}=T_{n \bmod N}$, taking values in $\{1,2, \ldots, N\}, \mu \in\left(0, \frac{2 \eta}{k^{2}}\right)$ and $\left\{\lambda_{n}\right\}$ is a sequence of real numbers in $(0,1)$, and proved that, under the following conditions:

(L1) $\lim _{n \rightarrow \infty} \lambda_{n}=0$,

(L2) $\sum_{n=1}^{\infty} \lambda_{n}=\infty$,

(L3) $\sum_{n=1}^{\infty}\left|\lambda_{n}-\lambda_{n+N}\right|<\infty$, and

(L4) $C=\operatorname{Fix}\left(T_{1} T_{2} \cdots T_{N-1} T_{N}\right)=\operatorname{Fix}\left(T_{N} T_{1} \cdots T_{N-2} T_{N-1}\right)=\cdots=\operatorname{Fix}\left(T_{2} T_{3} \cdots T_{N} T_{1}\right)$,

the sequence $\left\{x_{n}\right\}$ generated by (1.5) converges strongly to a unique solution of (1.2). The algorithms and convergence results of Yamada in [7] have been improved and extended to a finite or an infinite family of nonexpansive mappings; see, for example, $\mathrm{Xu}$ and Kim [8], Zeng [9], Liu and Cai [10], and Iemoto and Takahashi [11]. However, all such improvements and extensions are confined to a finite or an infinite family of nonexpansive mappings.

In this paper, we propose a new iterative algorithm based on a combination of the projected gradient method for variational inequalities with the Ishikawa-type method for fixed point problems to solve (1.2) with $C=\bigcap_{i=1}^{N} \operatorname{Fix}\left(T_{i}\right)$, where $\left\{T_{i}\right\}_{i=1}^{N}$ is a finite family of $L_{i}$-Lipschitz continuous and quasi-pseudocontractive mappings on $\Omega$, where $\Omega$ is a nonempty closed and convex subset of $H$, while $F: \Omega \rightarrow H$ is a $k$-Lipschitz continuous and $\eta$-strongly monotone mapping.

Given a stating point $x_{1} \in \Omega$, the iteration is generated by

$$
\left\{\begin{array}{l}
x_{1} \in \Omega, \\
y_{n i}=\left(1-\alpha_{n i}\right) x_{n}+\alpha_{n i} T_{i} x_{n}, \quad i=1,2, \ldots, N, \\
x_{n+1}=P_{\Omega}\left[\left(I-\lambda_{n} \mu F\right)\left(\beta_{n 0} x_{n}+\sum_{i=1}^{N} \beta_{n i} T_{i} y_{n i}\right)\right], \quad n \geq 1,
\end{array}\right.
$$


where $\left\{\beta_{n i}: i=0,1,2, \ldots, N\right\} \subset(a, b) \subset(0,1),\left\{\lambda_{n}\right\} \subset(0,1)$ satisfy the following conditions:

(i) $\sum_{i=0}^{N} \beta_{n i}=1$;

(ii) $\lim _{n \rightarrow \infty} \lambda_{n}=0, \sum_{n=1}^{\infty} \lambda_{n}=\infty$;

(iii) $\sum_{i=1}^{N} \beta_{n i} \leq \min \left\{\alpha_{n i}: i=1,2, \ldots, N\right\} \leq \max \left\{\alpha_{n i}: i=1,2\right\} \leq \alpha<\frac{1}{\sqrt{1+L^{2}}+1}, \forall n \geq 1$, where $L:=\max \left\{L_{i}: i=1,2, \ldots, N\right\}$, while $\mu$ is a fixed constant satisfying $\mu \in\left(0, \frac{2 \eta}{k^{2}}\right)$.

By virtue of new analysis techniques, we prove that the sequence generated by (1.6) converges strongly to a unique solution of (1.2) with $C=\bigcap_{i=1}^{N} \operatorname{Fix}\left(T_{i}\right)$.

In order to reach our goal, we need the following conceptions and facts.

Let $D$ be a nonempty subset of a real Hilbert space $H$. A mapping $T: D \rightarrow H$ is called $\kappa$-strictly pseudocontractive if and only if there exists a constant $\kappa \in[0,1)$ such that

$$
\|T x-T y\|^{2} \leq\|x-y\|^{2}+\kappa\|(I-T) x-(I-T) y\|^{2}
$$

for all $x, y \in D$. When $\kappa=1, T$ is said to be pseudocontractive.

$T$ is said to be quasi-pseudocontractive if and only if $\operatorname{Fix}(T) \neq \varnothing$ and

$$
\|T x-y\|^{2} \leq\|x-y\|^{2}+\|(I-T) x\|^{2}
$$

for all $x \in D$ but $y \in \operatorname{Fix}(T)$.

We remark that inequalities (1.7) and (1.8) are equivalent to the inequalities

$$
\langle T x-T y, x-y\rangle \leq\|x-y\|^{2}-\frac{1-\kappa}{2}\|(I-T) x-(I-T) y\|^{2} \quad \text { for all } x, y \in D
$$

and

$$
\langle T x-y, x-y\rangle \leq\|x-y\|^{2}
$$

for all $x \in D$ but $y \in \operatorname{Fix}(T)$, respectively.

We note that if $T$ is $\kappa$-strictly pseudocontractive, then it is Lipschitz continuous and pseudocontractive; if $T$ is a pseudocontraction with a fixed point, then $T$ is a quasipseudocontraction; however, the converse may be not true.

Recall that the metric (nearest point) projection from $H$ onto a nonempty closed convex subset $E$ of $H$ is defined as follows: for each point $x \in H$, there exists a unique point $P_{E} x \in E$ with the property

$$
\left\|x-P_{E} x\right\| \leq\|x-y\| \quad \text { for all } y \in E,
$$

that is, for any point $x \in H, \bar{x}=P_{E} x$ if and only if $\bar{x} \in E$ and $\|x-\bar{x}\|=\inf \{\|x-y\|: y \in E\}$.

Lemma 1.1 [12,13] Let $P_{E}: H \rightarrow E$ be a metric projection from $H$ on a nonempty closed convex subset $E$ of $H$. Then the following conclusions hold true:

$\left(\mathrm{p}_{1}\right)$ Given $x \in H$ and $z \in E$. Then $z=P_{E} x$ if and only if there holds the inequality

$$
\langle x-z, y-z\rangle \leq 0, \quad \forall y \in E .
$$


$\left(\mathrm{p}_{2}\right)$

$$
\left\langle P_{E} x-P_{E} y, x-y\right\rangle \geq\left\|P_{E} x-P_{E} y\right\|^{2}, \quad \forall x, y \in H,
$$

in particular, one has

$$
\left\|P_{E} x-P_{E} y\right\| \leq\|x-y\|, \quad \forall x, y \in H .
$$

Lemma $1.2[14,15]$

(i) $\|x \pm y\|^{2}=\|x\|^{2} \pm 2\langle x, y\rangle+\|y\|^{2}$ for all $x, y \in H$;

(ii) $\|(1-t) x+t y\|^{2}=(1-t)\|x\|^{2}+t\|y\|^{2}-t(1-t)\|x-y\|^{2}$ for all $x, y \in H$ and $t \in \mathbb{R}$;

(iii) for all $x_{i} \in H$ and $\alpha_{i} \in[0,1](i=1,2, \ldots, n)$ such that $\sum_{i=1}^{n} \alpha_{i}=1$, the following equality holds:

$$
\left\|\sum_{i=1}^{n} \alpha_{i} x_{i}\right\|^{2}=\sum_{i=1}^{n} \alpha_{i}\left\|x_{i}\right\|^{2}-\sum_{1 \leq i<j \leq n} \alpha_{i} \alpha_{j}\left\|x_{i}-x_{j}\right\|^{2} .
$$

Lemma 1.3 [7] Let $\Omega$ be a nonempty subset of $H$ and $F: \Omega \rightarrow H$ be a k-Lipschitz continuous and $\eta$-strongly monotone mapping. For each $\lambda \in(0,1]$ and $\mu \in\left(0, \frac{2 \eta}{k^{2}}\right)$, write $T^{\lambda}:=(I-\lambda \mu F)$ and $\tau:=1-\sqrt{1-\mu\left(2 \eta-\mu k^{2}\right)} \in(0,1)$. Then we have

$$
\left\|T^{\lambda} x-T^{\lambda} y\right\| \leq(1-\lambda \tau)\|x-y\|
$$

for all $x, y \in \Omega$.

Lemma 1.4 [16] Let E be a nonempty closed convex subset of a real Hilbert space $H$ and $T$ : $E \rightarrow E$ be L-Lipschitz continuous and quasi-pseudocontractive. Then $\operatorname{Fix}(T)$ is a nonempty closed convex subset of $E$, and therefore $P_{\mathrm{Fix}(T)} x$ is well defined for each $x \in H$.

Lemma 1.5 [17] Let E be a nonempty closed convex subset of a real Hilbert space $H$ and $T: E \rightarrow$ E be a demicontinuous pseudocontraction from $E$ into itself. Then $\operatorname{Fix}(T)$ is a closed convex subset of $E$ and $I-T$ is demiclosed at zero.

Lemma 1.6 [18] Let $\left\{a_{n}\right\}$ be a sequence of real numbers such that there exists a subsequence $\left\{n_{i}\right\}$ of $\{n\}$ such that $a_{n_{i}}<a_{n_{i}+1}$ for all $i \in \mathbb{N}$. Then there exists a nondecreasing sequence $\left\{m_{k}\right\} \subset \mathbb{N}$ such that $m_{k} \rightarrow \infty$ and the following properties are fulfilled:

$$
a_{m_{k}} \leq a_{m_{k}+1} \quad \text { and } \quad a_{k} \leq a_{m_{k}+1}
$$

for all sufficiently large numbers $k \in \mathbb{N}$.

Lemma 1.7 [19] Let $\left\{s_{n}\right\}$ be a sequence of nonnegative real numbers satisfying the following relation:

$$
s_{n+1} \leq\left(1-t_{n}\right) s_{n}+t_{n} \sigma_{n}, \quad n \geq n_{0},
$$

where $\left\{t_{n}\right\} \subset(0,1)$ and $\left\{\sigma_{n}\right\} \subset R$ satisfy the following conditions: $\lim _{n \rightarrow \infty} t_{n}=0, \sum_{n=1}^{\infty} t_{n}=$ $\infty$, and $\varlimsup_{n \rightarrow \infty} \sigma_{n} \leq 0$. Then $s_{n} \rightarrow 0$ as $n \rightarrow \infty$. 


\section{Main results}

Theorem 2.1 Let $\Omega$ be a nonempty, closed and convex subset of a real Hilbert space $H$. Let $T_{1}, T_{2}, \ldots, T_{N}: \Omega \rightarrow \Omega$ be $L_{i}$-Lipschitz continuous and quasi-pseudocontractive with Lipschitz constants $L_{1}, L_{2}, \ldots, L_{N}$, respectively. Let $F: \Omega \rightarrow H$ be a k-Lipschitz continuous and $\eta$-strongly monotone mapping. Assume that $\mathcal{F}=\bigcap_{i=1}^{N} \operatorname{Fix}\left(T_{i}\right) \neq \varnothing$ and $I-T_{i}$ are demiclosed at zero for $i=1,2, \ldots, N$. Let $\left\{x_{n}\right\}$ be defined by (1.6). Then $\left\{x_{n}\right\}$ converges strongly to a unique solution $x^{*}$ of $(1.2)$, where $x^{*}=P_{\mathcal{F}}(I-\mu F) x^{*}$.

Proof First of all, we show that $P_{\mathcal{F}} x$ is well defined for each $x \in H$. Indeed, in view of Lemma 1.4, we know that $\operatorname{Fix}\left(T_{i}\right)$ are closed convex for $i=1,2, \ldots, N$, and hence $\mathcal{F}$ is also nonempty, closed and convex; consequently, $P_{\mathcal{F}} x$ is well defined for any $x \in H$. Secondly, we show that there exists a unique $x^{*} \in \mathcal{F}$ such that

$$
x^{*}=P_{\mathcal{F}}(I-\mu F) x^{*} .
$$

Indeed, in view of Lemma 1.3, we know that $I-\mu F: \Omega \rightarrow H$ is a contraction, and hence $P_{\mathcal{F}}(I-\mu F): \Omega \rightarrow \Omega$ is also a contraction on $\Omega$. Then we use the Banach contraction mapping principle to deduce (2.1).

Write $u_{n}=\beta_{n 0} x_{n}+\sum_{i=1}^{N} \beta_{n i} T_{i} y_{n i}$. Then, $\forall p \in \mathcal{F}$, by virtue of Lemma 1.2, (1.6) and (1.8), we have that

$$
\begin{aligned}
&\left\|y_{n i}-p\right\|^{2} \\
&=\left\|\left(1-\alpha_{n i}\right)\left(x_{n}-p\right)+\alpha_{n i}\left(T_{i} x_{n}-p\right)\right\|^{2} \\
&=\left(1-\alpha_{n i}\right)\left\|x_{n}-p\right\|^{2}+\alpha_{n i}\left\|T_{i} x_{n}-p\right\|^{2}-\alpha_{n i}\left(1-\alpha_{n i}\right)\left\|x_{n}-T_{i} x_{n}\right\|^{2} \\
& \leq\left(1-\alpha_{n i}\right)\left\|x_{n}-p\right\|^{2}+\alpha_{n i}\left[\left\|x_{n}-p\right\|^{2}+\left\|x_{n}-T_{i} x_{n}\right\|^{2}\right] \\
&-\alpha_{n i}\left(1-\alpha_{n i}\right)\left\|x_{n}-T_{i} x_{n}\right\|^{2} \\
&=\left\|x_{n}-p\right\|^{2}+\alpha_{n i}^{2}\left\|x_{n}-T_{i} x_{n}\right\|^{2}
\end{aligned}
$$

for $i=1,2, \ldots, N$ and all $n \geq 1$.

Furthermore, from (1.6) and Lemma 1.2, we get that

$$
\begin{aligned}
\| y_{n i} & -T_{i} y_{n i} \|^{2} \\
& =\left\|\left(1-\alpha_{n i}\right)\left(x_{n}-T_{i} y_{n i}\right)+\alpha_{n i}\left(T_{i} x_{n}-T_{i} y_{n i}\right)\right\|^{2} \\
& =\left(1-\alpha_{n i}\right)\left\|x_{n}-T_{i} y_{n i}\right\|^{2}+\alpha_{n i}\left\|T_{i} x_{n}-T_{i} y_{n i}\right\|^{2}-\alpha_{n i}\left(1-\alpha_{n i}\right)\left\|x_{n}-T_{i} x_{n}\right\|^{2} \\
& \leq\left(1-\alpha_{n i}\right)\left\|x_{n}-T_{i} y_{n i}\right\|^{2}+\alpha_{n i} L^{2}\left\|x_{n}-y_{n i}\right\|^{2}-\alpha_{n i}\left(1-\alpha_{n i}\right)\left\|x_{n}-T_{i} x_{n}\right\|^{2} \\
& =\left(1-\alpha_{n i}\right)\left\|x_{n}-T_{i} y_{n i}\right\|^{2}+\alpha_{n i}^{3} L^{2}\left\|x_{n}-T_{i} x_{n}\right\|^{2}-\alpha_{n i}\left(1-\alpha_{n i}\right)\left\|x_{n}-T_{i} x_{n}\right\|^{2} \\
& =\left(1-\alpha_{n i}\right)\left\|x_{n}-T_{i} y_{n i}\right\|^{2}-\alpha_{n i}\left(1-\alpha_{n i}-L^{2} \alpha_{n i}^{2}\right)\left\|x_{n}-T_{i} x_{n}\right\|^{2}
\end{aligned}
$$

for $i=1,2, \ldots, N$ and all $n \geq 1$. 
At this point, we can estimate $\left\|u_{n}-p\right\|^{2}$. In fact, from Lemma 1.2, (2.2), (2.3) and conditions (i) and (iii) in (1.6), we have

$$
\begin{aligned}
& \left\|u_{n}-p\right\|^{2} \\
& =\left\|\beta_{n 0}\left(x_{n}-p\right)+\sum_{i=1}^{N} \beta_{n i}\left(T_{i} y_{n i}-p\right)\right\|^{2} \\
& =\beta_{n 0}\left\|x_{n}-p\right\|^{2}+\sum_{i=1}^{N} \beta_{n i}\left\|\left(T_{i} y_{n i}-p\right)\right\|^{2}-\sum_{i=1}^{N} \beta_{n 0} \beta_{n i}\left\|x_{n}-T_{i} y_{n i}\right\|^{2} \\
& -\sum_{1 \leq i<j \leq N} \beta_{n i} \beta_{n_{j}}\left\|T_{i} y_{n i}-T_{j} y_{n_{j}}\right\|^{2} \\
& \leq \beta_{n 0}\left\|x_{n}-p\right\|^{2}+\sum_{i=1}^{N} \beta_{n i}\left[\left\|y_{n i}-p\right\|^{2}+\left\|y_{n i}-T_{i} y_{n i}\right\|^{2}\right] \\
& -\sum_{i=1}^{N} \beta_{n 0} \beta_{n i}\left\|x_{n}-T_{i} y_{n i}\right\|^{2}-\sum_{1 \leq i<j \leq N} \beta_{n i} \beta_{n_{j}}\left\|T_{i} y_{n i}-T_{j} y_{n_{j}}\right\|^{2} \\
& \leq \beta_{n 0}\left\|x_{n}-p\right\|^{2}+\sum_{i=1}^{N} \beta_{n i}\left\|x_{n}-p\right\|^{2}+\sum_{i=1}^{N} \beta_{n i}\left(1-\alpha_{n i}\right)\left\|x_{n}-T_{i} y_{n i}\right\|^{2} \\
& -\sum_{i=1}^{N} \beta_{n i} \alpha_{n i}\left(1-\alpha_{n i}-L^{2} \alpha_{n i}^{2}\right)\left\|x_{n}-T_{i} x_{n}\right\|^{2}-\sum_{i=1}^{N} \beta_{n 0} \beta_{n i}\left\|x_{n}-T_{i} y_{n i}\right\|^{2} \\
& =\left\|x_{n}-p\right\|^{2}-\sum_{i=1}^{N} \beta_{n i} \alpha_{n i}\left(1-\alpha_{n i}-L^{2} \alpha_{n i}^{2}\right)\left\|x_{n}-T_{i} x_{n}\right\|^{2} \\
& +\sum_{i=1}^{N} \beta_{n i}\left(1-\alpha_{n i}-\beta_{n 0}\right)\left\|x_{n}-T_{i} y_{n i}\right\|^{2} \\
& \leq\left\|x_{n}-p\right\|^{2}-\sum_{i=1}^{N} \beta_{n i} \alpha_{n i}\left(1-\alpha_{n i}-L^{2} \alpha_{n i}^{2}\right)\left\|x_{n}-T_{i} x_{n}\right\|^{2} \\
& \leq\left\|x_{n}-p\right\|^{2}-\left(\sum_{i=1}^{N} \beta_{n i}\right)^{2}\left(1-\alpha-L^{2} \alpha^{2}\right)\left\|x_{n}-T_{i} x_{n}\right\|^{2} \\
& \leq\left\|x_{n}-p\right\|^{2}-(N a)^{2}\left(1-\alpha-L^{2} \alpha^{2}\right)\left\|x_{n}-T_{i} x_{n}\right\|^{2}
\end{aligned}
$$

for all $i=1,2, \ldots, N$ and all $n \geq 1$.

Note that $1-\alpha-L^{2} \alpha^{2}>0$, it follows from (2.4) that

$$
\left\|u_{n}-p\right\| \leq\left\|x_{n}-p\right\| \quad \text { for all } n \geq 1
$$

In particular, for $x^{*}=P_{\mathcal{F}}(I-\mu F) x^{*} \in \mathcal{F}$, we have

$$
\left\|u_{n}-x^{*}\right\| \leq\left\|x_{n}-x^{*}\right\| \quad \text { for all } n \geq 1
$$


From Lemmas 1.1, 1.3 and (2.6), we can prove that $\left\{x_{n}\right\}$ is bounded. Indeed, we have

$$
\begin{aligned}
\left\|x_{n+1}-x^{*}\right\| & =\left\|P_{\Omega}\left[\left(I-\lambda_{n} \mu F\right) u_{n}\right]-x^{*}\right\|=\left\|P_{\Omega}\left[\left(I-\lambda_{n} \mu F\right) u_{n}\right]-P_{\Omega} x^{*}\right\| \\
& \leq\left\|\left(I-\lambda_{n} \mu F\right) u_{n}-x^{*}\right\| \\
& =\left\|\left(I-\lambda_{n} \mu F\right) u_{n}-\left(I-\lambda_{n} \mu F\right) x^{*}-\lambda_{n} \mu F x^{*}\right\| \\
& =\left\|\left(I-\lambda_{n} \mu F\right) u_{n}-\left(I-\lambda_{n} \mu F\right) x^{*}\right\|+\lambda_{n} \mu\left\|F x^{*}\right\| \\
& \leq\left(1-\tau \lambda_{n}\right)\left\|u_{n}-x^{*}\right\|+\mu \lambda_{n}\left\|F x^{*}\right\| \\
& \leq\left(1-\tau \lambda_{n}\right)\left\|u_{n}-x^{*}\right\|+\tau \lambda_{n} \frac{\mu\left\|F x^{*}\right\|}{\tau} \\
& \leq\left(1-\tau \lambda_{n}\right)\left\|x_{n}-x^{*}\right\|+\tau \lambda_{n} \frac{\mu\left\|F x^{*}\right\|}{\tau} \\
& \leq \max \left\{\left\|x_{1}-x^{*}\right\|, \frac{\mu\left\|F x^{*}\right\|}{\tau}\right\}:=M
\end{aligned}
$$

for all $n \geq 1$, and therefore $\left\{x_{n}\right\}$ is bounded; consequently, $\left\{y_{n i}\right\},\left\{u_{n}\right\}$ and $\left\{F u_{n}\right\}$ are all bounded.

We next show that $x_{n} \rightarrow x^{*}(n \rightarrow \infty)$.

By virtue of Lemmas 1.1-1.3, (1.6) and (2.4), we have that

$$
\begin{aligned}
\left\|x_{n+1}-x^{*}\right\|^{2}= & \left\|P_{\Omega}\left[\left(I-\lambda_{n} \mu F\right) u_{n}\right]-x^{*}\right\|^{2} \leq\left\|\left(I-\lambda_{n} \mu F\right) u_{n}-x^{*}\right\|^{2} \\
= & \left\|\left(I-\lambda_{n} \mu F\right) u_{n}-\left(I-\lambda_{n} \mu F\right) x^{*}-\lambda_{n} \mu F x^{*}\right\|^{2} \\
= & \left\|\left(I-\lambda_{n} \mu F\right) u_{n}-\left(I-\lambda_{n} \mu F\right) x^{*}\right\|^{2}+\lambda_{n}^{2} \mu^{2}\left\|F x^{*}\right\|^{2} \\
& +2 \mu \lambda_{n}\left\langle F x^{*},\left(I-\lambda_{n} \mu F\right) u_{n}-\left(I-\lambda_{n} \mu F\right) x^{*}\right\rangle \\
\leq & \left(1-\tau \lambda_{n}\right)\left\|u_{n}-x^{*}\right\|^{2}+\lambda_{n}^{2} \mu^{2}\left\|F x^{*}\right\|^{2} \\
& +2 \mu \lambda_{n}\left\langle F x^{*}, x^{*}-u_{n}\right\rangle+2 \mu^{2} \lambda_{n}^{2}\left\langle F x^{*}, F u_{n}-F x^{*}\right\rangle \\
= & \left(1-\tau \lambda_{n}\right)\left[\left\|x_{n}-x^{*}\right\|^{2}-(N a)^{2}\left(1-\alpha-L^{2} \alpha^{2}\right)\left\|x_{n}-T_{i} x_{n}\right\|^{2}\right] \\
& +2 \mu \lambda_{n}\left\langle F x^{*}, x^{*}-u_{n}\right\rangle+2 \mu^{2} \lambda_{n}^{2}\left\langle F x^{*}, F u_{n}\right\rangle-\lambda_{n}^{2} \mu^{2}\left\|F x^{*}\right\|^{2} \\
\leq & \left(1-\tau \lambda_{n}\right)\left\|x_{n}-x^{*}\right\|^{2}-\left(1-\tau \lambda_{n}\right) C_{1}\left\|x_{n}-T_{i} x_{n}\right\|^{2} \\
& +2 \mu \lambda_{n}\left\langle F x^{*}, x^{*}-u_{n}\right\rangle+2 \mu^{2}\left\|F x^{*}\right\| \lambda_{n}^{2}\left\|F u_{n}\right\| \\
\leq & \left(1-\tau \lambda_{n}\right)\left\|x_{n}-x^{*}\right\|^{2}-\left(1-\tau \lambda_{n}\right) C_{1}\left\|x_{n}-T_{i} x_{n}\right\|^{2} \\
& +2 \mu \lambda_{n}\left\langle F x^{*}, x^{*}-u_{n}\right\rangle+C_{2} \lambda_{n}^{2}
\end{aligned}
$$

for $i=1,2, \ldots, N$ and all $n \geq 1$, where $C_{1}=(N a)^{2}\left(1-\alpha-L^{2} \alpha^{2}\right)$ and $C_{2}=2 \mu^{2}\left\|F x^{*}\right\| \times$ $\sup \left\{\left\|F u_{n}\right\|: n \geq 1\right\}$ are fixed positive constants.

Set $s_{n}=\left\|x_{n}-x^{*}\right\|^{2}$. Then (2.7) reduces to

$$
s_{n+1}-s_{n}+\tau \lambda_{n} s_{n}+\left(1-\tau \lambda_{n}\right) C_{1}\left\|x_{n}-T_{i} x_{n}\right\|^{2} \leq 2 \mu \lambda_{n}\left\langle F x^{*}, x^{*}-u_{n}\right\rangle+C_{2} \lambda_{n}^{2}
$$

for $i=1,2, \ldots, N$ and all $n \geq 1$.

Now we consider two possible cases. 
Case $1 .\left\{s_{n}\right\}$ is decreasing eventually, that is, there exists some integer $n_{0} \geq 1$ such that

$$
s_{n+1} \leq s_{n} \quad \text { for all } n \geq n_{0} .
$$

In this case, we have $\lim _{n \rightarrow \infty} s_{n}$ exists.

Taking the limit in (2.8), noting that $\lambda_{n} \rightarrow 0$ as $n \rightarrow \infty$, we get that

$$
x_{n}-T_{i} x_{n} \rightarrow 0 \quad \text { as } n \rightarrow \infty
$$

for $i=1,2, \ldots, N$. It follows from (1.6) that

$$
x_{n}-y_{n i} \rightarrow 0 \quad \text { as } n \rightarrow \infty
$$

for $i=1,2, \ldots, N$. Since $T_{i}$ is $L_{i}$-Lipschitz continuous, we have that

$$
\left\|T_{i} x_{n}-T_{i} y_{n i}\right\| \leq L_{i}\left\|x_{n}-y_{n i}\right\|
$$

for $i=1,2, \ldots, N$. Consequently, from (2.9) (2.11) we get that

$$
\begin{aligned}
\left\|u_{n}-x_{n}\right\| & =\left\|\sum_{i=1}^{N} \beta_{n i}\left(x_{n}-T_{i} y_{n i}\right)\right\| \\
& \leq \sum_{i=1}^{N} \beta_{n i}\left\|x_{n}-T_{i} y_{n i}\right\| \\
& \leq \sum_{i=1}^{N} \beta_{n i}\left\|x_{n}-T_{i} x_{n}\right\|+\sum_{i=1}^{N} \beta_{n i}\left\|T_{i} x_{n}-T_{i} y_{n i}\right\| \\
& \leq b \sum_{i=1}^{N}\left\|x_{n}-T_{i} x_{n}\right\|+L b \sum_{i=1}^{N}\left\|x_{n}-y_{n i}\right\| \rightarrow 0
\end{aligned}
$$

as $n \rightarrow \infty$, which derives that

$$
\varlimsup_{n \rightarrow \infty}\left\langle F x^{*}, x^{*}-u_{n}\right\rangle=\varlimsup_{n \rightarrow \infty}\left\langle F x^{*}, x^{*}-x_{n}\right\rangle .
$$

Assume that

$$
\varlimsup_{n \rightarrow \infty}\left\langle F x^{*}, x^{*}-x_{n}\right\rangle=\lim _{k \rightarrow \infty}\left\langle F x^{*}, x^{*}-x_{n k}\right\rangle .
$$

Without loss of generality, we can assume that $x_{n k} \rightarrow \hat{x}$ weakly as $k \rightarrow \infty$; then $\hat{x}=T_{i} \hat{x}$ for $i=1,2, \ldots, N$, by virtue of (2.9) and our assumption, and hence $\hat{x} \in \mathcal{F}$. It follows from (2.13) and (1.2) that

$$
\varlimsup_{n \rightarrow \infty}\left\langle F x^{*}, x^{*}-u_{n}\right\rangle=\left\langle F x^{*}, x^{*}-\hat{x}\right\rangle \leq 0 .
$$

Set $t_{n}=\tau \lambda_{n}$ and $\sigma_{n}=\frac{2 \mu}{\tau}\left\langle F x^{*}, x^{*}-u_{n}\right\rangle+\frac{C_{2}}{\tau} \lambda_{n}$. Then (2.8) reduces to

$$
s_{n+1} \leq\left(1-t_{n}\right) s_{n}+t_{n} \sigma_{n},
$$

where $\varlimsup_{n \rightarrow \infty} \sigma_{n} \leq 0$. Now Lemma 1.7 can be used to deduce $s_{n} \rightarrow 0$ as $n \rightarrow \infty$. 
Case 2. $\left\{s_{n}\right\}$ is not decreasing eventually, that is, there exists a subsequence $\{n i\}$ of $\{n\}$ such that $s_{n i} \leq s_{n i+1}$ for all $i \in \mathbb{N}$. By virtue of Lemma 1.6, we know that there exists a nondecreasing sequence $\left\{m_{k}\right\} \subset \mathbb{N}$ such that $m_{k} \rightarrow \infty, s_{m_{k}} \leq s_{m_{k}+1}$ and $s_{k} \leq s_{m_{k}+1}$ for all sufficiently large $k \in \mathbb{N}$. In this case, we have $s_{m_{k}+1}-s_{m_{k}} \geq 0$ for large enough $k \in \mathbb{N}$. It follows from (2.8) that

$$
\begin{aligned}
& \lim _{k \rightarrow \infty}\left(s_{m_{k}+1}-s_{m_{k}}\right)=0, \\
& \lim _{k \rightarrow \infty}\left(x_{m_{k}}-T_{i} x_{m_{k}}\right)=0 \quad \text { for } i=1,2, \ldots, N,
\end{aligned}
$$

and

$$
\varlimsup_{k \rightarrow \infty} s_{m_{k}} \leq 2 \mu \varlimsup_{k \rightarrow \infty}\left\langle F x^{*}, x^{*}-u_{m_{k}}\right\rangle .
$$

By using a reasoning similar to case 1 , we can obtain that $\varlimsup_{k \rightarrow \infty}\left\langle F x^{*}, x^{*}-u_{m_{k}}\right\rangle \leq 0$, and hence $\varlimsup_{k \rightarrow \infty} s_{m_{k}} \leq 0$ by (2.17), i.e., $s_{m_{k}} \rightarrow 0$ as $k \rightarrow \infty$, which derives $s_{m_{k}+1} \rightarrow 0$ as $k \rightarrow \infty$; consequently, $s_{k} \rightarrow 0$ as $k \rightarrow \infty$, since $s_{k} \leq s_{m_{k}+1}$ for sufficiently large $k \in \mathbb{N}$. This completes the proof.

Remark 2.1 When $\Omega=H, P_{\Omega}$ in (1.6) can be dropped.

Corollary 2.1 Let $\Omega$ be a nonempty, closed and convex subset of a real Hilbert space $H$. Let $T_{1}, T_{2}, \ldots, T_{N}: \Omega \rightarrow \Omega$ be $N L_{i}$-Lipschitz continuous and strongly pseudocontractive with Lipschitz constants $L_{1}, L_{2}, \ldots, L_{N}$, respectively. Let $F, \mathcal{F}$ and $\left\{x_{n}\right\}$ be the same as in Theorem 2.1. Then $\left\{x_{n}\right\}$ converges strongly to a unique solution $x^{*}$ of $(1.2)$, where $x^{*}=P_{\mathcal{F}}(I-$ $\mu F) x^{*}$.

Proof By virtue of Lemma 1.5, we know that $\operatorname{Fix}\left(T_{i}\right)$ are closed convex for $i=1,2, \ldots, N$ and hence $\mathcal{F}=\bigcap_{i=1}^{N} \operatorname{Fix}\left(T_{i}\right)$ is nonempty, closed and convex. Lemma 1.5 also ensures that $I-T_{i}$ are demiclosed at zero for $i=1,2, \ldots, N$ and hence the conclusion of Corollary 2.1 follows exactly from Theorem 2.1.

Corollary 2.2 Let $\Omega$ be a nonempty, closed and convex subset of a real Hilbert space $H$. Let $T_{1}, T_{2}, \ldots, T_{N}: \Omega \rightarrow \Omega$ be $N$ strict pseudocontractions, respectively. Let $F, \mathcal{F}$ and $\left\{x_{n}\right\}$ be the same as in Theorem 2.1. Then $\left\{x_{n}\right\}$ converges strongly to a unique solution $x^{*}$ of (1.2), where $x^{*}=P_{\mathcal{F}}(I-\mu F) x^{*}$.

Proof Since every strictly pseudocontractive mapping is Lipschitz continuous and pseudocontractive, we have the desired conclusion.

Corollary 2.3 Let $\Omega$ be a nonempty, closed and convex subset of a real Hilbert space $H$. Let $T_{1}, T_{2}, \ldots, T_{N}: \Omega \rightarrow \Omega$ be $N$ nonexpansive mappings, respectively. Let $F, \mathcal{F}$ and $\left\{x_{n}\right\}$ be the same as in Theorem 2.1. Then $\left\{x_{n}\right\}$ converges strongly to a unique solution $x^{*}$ of (1.2), where $x^{*}=P_{\mathcal{F}}(I-\mu F) x^{*}$.

Proof Since any nonexpansive mapping is 1-Lipschitz continuous and pseudocontractive, we have the desired conclusion by Corollary 2.2 . 
Table 1 The results of the algorithm in [20]

\begin{tabular}{lllllllll}
\hline $\boldsymbol{n}$ & $\mathbf{0}$ & $\mathbf{5 0 0}$ & $\mathbf{1 , 0 0 0}$ & $\mathbf{5 , 0 0 0}$ & $\mathbf{1 0 , 0 0 0}$ & $\mathbf{1 2 , 0 0 0}$ & $\mathbf{1 4 , 0 0 0}$ & $\mathbf{1 7 , 0 0 0}$ \\
\hline$x_{1}$ & 2 & 1.8602 & 1.8441 & 1.8074 & 1.7919 & 1.7878 & 1.7843 & 1.7800 \\
$x_{2}$ & 3 & 2.7902 & 2.7662 & 2.7112 & 2.6878 & 2.6817 & 2.6765 & 2.6700 \\
\hline
\end{tabular}

Table 2 The results of algorithm (1.6)

\begin{tabular}{lllllllll}
\hline $\boldsymbol{n}$ & $\mathbf{0}$ & $\mathbf{5 0 0}$ & $\mathbf{1 , 0 0 0}$ & $\mathbf{5 , 0 0 0}$ & $\mathbf{1 0 , 0 0 0}$ & $\mathbf{1 2 , 0 0 0}$ & $\mathbf{1 4 , 0 0 0}$ & $\mathbf{1 7 , 0 0 0}$ \\
\hline$x_{1}$ & 2 & 1.4957 & 1.4490 & 1.3332 & 1.2878 & 1.2761 & 1.2663 & 1.2541 \\
$x_{2}$ & 3 & 2.2435 & 2.1673 & 1.9998 & 1.9317 & 1.9142 & 1.8995 & 1.8812 \\
\hline
\end{tabular}

Remark 2.2 When $F=I$, we have the following strong convergence theorem.

Corollary 2.4 Let $\Omega$ be a nonempty, closed and convex subset of a real Hilbert space $H$. Let $T_{1}, T_{2}, \ldots, T_{N}: \Omega \rightarrow \Omega$ be $N L_{i}$-Lipschitz continuous and quasi-pseudocontractive with Lipschitz constants $L_{1}, L_{2}, \ldots, L_{N}$, respectively. Let $\mathcal{F}$ and $I-T_{i}$ be the same as in Theorem 2.1. Let $\left\{x_{n}\right\}$ be defined by (1.6) with $F=I$. Then $\left\{x_{n}\right\}$ converges strongly to the minimum-norm fixed point of the family $\left\{T_{i}\right\}_{i=1}^{N}$.

\section{Numerical example}

Example 3.1 [20] Consider the following optimization problem: find an element

$$
x^{*} \in C: \varphi\left(x^{*}\right)=\min _{x \in C} \varphi(x)
$$

where $\varphi(x)=\frac{1}{2}\|x\|^{2}, x=\left(x_{1}, x_{2}\right) \in \mathbb{R}^{2}$, a Euclid space, and $C=C_{1} \cap C_{2}$, defined by

$$
\begin{aligned}
& C_{1}=\left\{\left(x_{1}, x_{2}\right) \in \mathbb{R}^{2}: x_{1}-2 x_{2}+1 \leq 0\right\}, \\
& C_{2}=\left\{\left(x_{1}, x_{2}\right) \in \mathbb{R}^{2}: 4 x_{1}-x_{2}-3 \geq 0\right\}
\end{aligned}
$$

(3.1) has a unique solution $x^{*}=(1,1)$ and $F=\nabla \varphi=I$ is 1 -Lipschitz continuous and $\frac{1}{2}$ strongly monotone. Starting with the point $x^{1}=\left(x_{1}, x_{2}\right)=(2,3), \mu=\frac{1}{20} \in(0,1)$ and $\lambda_{n}=$ $\frac{1}{n+1}$, set $\alpha_{n i}=\frac{1}{100}+\frac{1}{n+100}, \beta_{n_{1}}=\beta_{n_{2}}=\frac{1}{2} \alpha_{n i}, \beta_{n 0}=1-\alpha_{n i}$ for $i=1,2$, Table 1 shows the results of algorithm in [20], we obtained the results of algorithm (1.6) in Table 2. Obviously, the results in Table 2 are better.

Example 3.2 [21] Let $H=\mathbb{R}$ with absolute value norm. Let $\Omega=[-2,1]$ and $T_{1}, T_{2}: \Omega \rightarrow \Omega$ be defined by

$$
T_{1} x= \begin{cases}x+x^{2}, & x \in[-2,0] \\ x, & x \in(0,1]\end{cases}
$$

and

$$
T_{2} x:= \begin{cases}x, & x \in\left[-2, \frac{1}{2}\right], \\ x-2\left(x-\frac{1}{2}\right)^{2}, & x \in\left(\frac{1}{2}, 1\right] .\end{cases}
$$


Table 3 Values of $\left\{x_{n}\right\}$ with initial values $x_{0}=-1$ and $x_{0}=0.8$

\begin{tabular}{rccrrrrrr}
\hline $\boldsymbol{n}$ & $\mathbf{0}$ & $\mathbf{5 0 0}$ & $\mathbf{1 , 0 0 0}$ & $\mathbf{5 , 0 0 0}$ & $\mathbf{1 0 , 0 0 0}$ & $\mathbf{1 2 , 0 0 0}$ & $\mathbf{1 4 , 0 0 0}$ & $\mathbf{1 7 , 0 0 0}$ \\
\hline$x_{n}$ & -1 & -0.0739 & -0.0447 & -0.0127 & -0.0071 & -0.0060 & -0.0053 & -0.0045 \\
$x_{n}$ & 0.8 & 0.1130 & 0.0803 & 0.0361 & 0.0255 & 0.0233 & 0.0216 & 0.0196 \\
\hline
\end{tabular}

Then $\mathcal{F}=\operatorname{Fix}\left(T_{1}\right) \cap \operatorname{Fix}\left(T_{2}\right)=[0,1] \cap\left[-2, \frac{1}{2}\right]=\left[0, \frac{1}{2}\right], T_{1}: \Omega \rightarrow \Omega$ is 5 -Lipschitz continuous and pseudocontractive and $T_{2}: \Omega \rightarrow \Omega$ is 10 -Lipschitz continuous and pseudocontractive. We find the point $x^{*} \in \mathcal{F}$ with the minimum-norm. To do so, set $F=I$.

Now, taking $\lambda_{n}=\frac{1}{n+10}, \alpha_{n i}=\frac{1}{100}+\frac{1}{n+100}, \beta_{n_{1}}=\beta_{n_{2}}=\frac{1}{2} \alpha_{n i}, \beta_{n 0}=1-\alpha_{n i}$ for $i=1,2$, and $\mu=$ $\frac{1}{2} \in\left(0, \frac{2 \eta}{k^{2}}\right)=(0,1)$, we see that the conditions of Corollary 2.1 are fulfilled and algorithm (1.6) provides the data in Table 3 . Our result is better.

\section{Competing interests}

The authors declare that they have no competing interests.

Authors' contributions

All the authors contributed equally.

\section{Author details}

${ }^{1}$ Department of Mathematics, Shijiazhuang Mechanical Engineering College, Shijiazhuang, 050003, China. ${ }^{2}$ Department of Mathematics and Information, Hebei Normal University, Shijiazhuang, 050016, China.

\section{Acknowledgements}

This research was supported by the National Natural Science Foundation of China (11071053).

Received: 14 November 2013 Accepted: 8 May 2014 Published: 30 May 2014

\section{References}

1. Kinderlehrer, D, Stampacchia, G: An Introduction to Variational Inequalities and Their Applications. Academic Press, New York (1980)

2. Glowinski, R: Numerical Methods for Nonlinear Variational Problems. Springer, New York (1984)

3. Zeidler, E: Nonlinear Functional Analysis Its Applications. Springer, New York (1985)

4. Goldstein, AA: Convex programming in Hilbert space. Bull. Am. Math. Soc. 70, 709-710 (1964)

5. Kassay, G, Reich, S, Sabach, S: Iterative methods for solving systems of variational inequalities in reflexive Banach spaces. SIAM J. Optim. 21, 1319-1344 (2011)

6. Censor, Y, Gibali, A, Reich, S, Sabach, S: Common solutions to variational inequalities. Set-Valued Var. Anal. 20, 229-247 (2012)

7. Yamada, Y: The hybrid steepest-descent method for variational inequality problems over the intersection of the fixed point sets of nonexpansive mappings. In: Butnariu, D, Censor, Y, Reich, S (eds.) Inherently Parallel Algorithms in Feasibility and Optimization and Their Application, pp. 473-504. North-Holland, Amsterdam (2001)

8. Xu, HK, Kim, TH: Convergence of hybrid steepest-descent methods for variational inequalities. J. Optim. Theory Appl. 119, 185-201 (2003)

9. Zeng, LC, Wong, NC, Yao, JC: Convergence analysis of modified hybrid steepest-descent methods with variable parameters for variational inequalities. J. Optim. Theory Appl. 132, 51-69 (2007)

10. Liu, X, Cui, Y: The common minimal-norm fixed point of a finite family of nonexpansive mappings. Nonlinear Anal. TMA 73, 76-83 (2010)

11. lemoto, S, Takahashi, W: Strong convergence theorems by a hybrid steepest descent method for countable nonexpansive mappings in Hilbert spaces. Sci. Math. Jpn. 21, 557-570 (2008)

12. Albert, Y: Metric and generalized projection operators in Banach spaces: properties and applications. In: Kartsatos, AG (ed.) Theory and Applications of Nonlinear Operators of Accretive and Monotone Type. Lecture Notes in Pure and Appl. Math., vol. 178, pp. 15-50. Dekker, Amsterdam (1996)

13. Goebel, K, Reich, S: Uniform Convexity, Hyperbolic Geometry, and Nonexpansive Mappings. Dekker, New York (1984)

14. Marino, G, Xu, HK: Weak and strong convergence theorems for strict pseudo-contractions in Hilbert spaces. J. Math. Anal. Appl. 329, 336-346 (2007)

15. Zegeye, H, Shahzad, N: Convergence of Mann's type iteration method for generalized asymptotically nonexpansive mappings. Comput. Math. Appl. 62, 4007-4014 (2011)

16. Zhou, HY, Su, YF: Strong convergence theorems for a family of quasi-pseudo-contractions in Hilbert spaces. Nonlinear Anal. 71, 120-125 (2009)

17. Zhou, HY: Convergence theorems of fixed points for Lipschitz pseudo-contractions in Hilbert spaces. J. Math. Anal. Appl. 343, 546-556 (2008)

18. Maingé, PE: Strong convergence of projected subgradient methods for nonsmooth and non-strictly convex minimization. Set-Valued Anal. 16, 899-912 (2008)

19. Xu, HK: Another control condition in an iterative method for nonexpansive mappings. Bull. Aust. Math. Soc. 65 109-113 (2002) 
20. Kim, JK, Buong, N: A new explicit iteration method for variational inequalities on the set of common fixed points for a finite family of nonexpansive mappings. J. Inequal. Appl. 2013, 419 (2013)

21. Zegeye, H, Shahzad, N: An algorithm for a common fixed point of a family of pseudocontractive mappings. Fixed Point Theory Appl. 2013, 234 (2013)

10.1186/1029-242X-2014-218

Cite this article as: Zhou and Wang: A new iteration method for variational inequalities on the set of common fixed points for a finite family of quasi-pseudocontractions in Hilbert spaces. Journal of Inequalities and Applications 2014, 2014:218

Submit your manuscript to a SpringerOpen ${ }^{\circ}$ journal and benefit from:

- Convenient online submission

- Rigorous peer review

- Immediate publication on acceptance

Open access: articles freely available online

- High visibility within the field

- Retaining the copyright to your article

Submit your next manuscript at $>$ springeropen.com 